\title{
Developing of Wire Rope Security Evaluation and Management System Wang Dongdong ${ }^{1, a, *}$, Liang Can ${ }^{1, b}$, Bai Wenjie ${ }^{1, c}$, Duan Quan ${ }^{1, d}$ \\ ${ }^{1}$ School of Chemical Engineering and Technology, Xi' an Jiaotong University, Xi' an, Shanxi,China \\ a'wang.123@stu.xjtu.edu.cn, b1138781295@qq.com, c490247779@qq.com, dQuanduan@mail.xjtu.edu.cn \\ ${ }^{*}$ Corresponding author
}

\begin{abstract}
On the basis of the running wire rope security and information management process, combined with the methods of acoustic emission nondestructive testing, security evaluation to running state of wire rope was realized. Moreover, wire rope information and users information were brought into management system to realize the informatization and high efficiency and make users information be under unified management. From the demand analysis and feasibility analysis of wire rope security evalution and management system, general design of the system was obtained, and then the system was developed by using Microsoft SQL Server 2000 as background database and visual programming tools Visual Basic 6.0. The system mainly includes login module, online evaluation module, wire rope information management module, users management module, data security module, software information module and help module, and could meet the needs of wire rope security evaluation and management after testing.
\end{abstract}

Keywords: wire rope, security evaluation, acoustic emission, database.

\author{
钢丝绳安全评价及管理系统开发

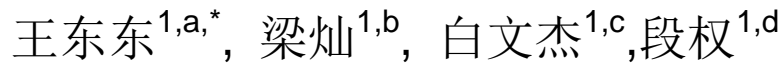 \\ ${ }^{1}$ 西安交通大学化学工程与技术学院, 西安, 陕西, 中国 \\ awang.123@stu.xjtu.edu.cn, b1138781295@qq.com, 490247779@qq.com, \\ 'Quanduan@mail.xjtu.edu.cn \\ *通讯作者
}

中文摘要. 基于钢丝绳运行中对安全及信 息的管理过程, 结合声发射无损检测方法, 实现对钢丝绳运行状况的安全评价; 同时, 将钢丝绳信息、用户信息纳入管理体系, 实 现对钢丝绳管理的信息化、高效化, 以及对 用户信息的统一管理。通过对钢丝绳安全评
价及管理系统进行需求分析和可行性分析, 进一步完成了系统的总体设计, 利用 Microsoft SQL Server 2000 作为后台数据 库, 结合Windows下可视化编程工具Visual Basic 6.0进行了系统开发。系统主要包括登 录、在线评价、钢丝绳信息管理、用户管理、 数据安全、软件信息、帮助模块, 对系统进 
行测试后, 可以满足钢丝绳安全评价及管理 工作的需要。

关键词: 钢丝绳; 安全评价; 声发射; 数 据库

\section{1. 引言}

在石油化工、交通运输、治金、煤炭、 港口、旅游架空索道与缆车等行业和部门, 钢丝绳作为承载、牵引过程中的重要部件, 发挥着必不可少的作用 ${ }^{[1]}$ 。然而, 由于在使 用过程中受到各种因素的影响, 钢丝绳会出 现不同程度的腐蚀、磨损、断丝等现象, 使 其使用强度降低, 甚至发生断裂 ${ }^{[2]}$ 。它的承 载能力和损伤情况会对生产的有序进行和 人生财产的安全产生直接的影响。因此, 实 现对使用中的钢丝绳断丝现象进行有效的 安全监测意义重大。目前, 主要采取定期“眼 观”、“手摸” 等办法来检查钢丝绳的状态, 或采用强制性定期更换钢丝绳的办法来保 证安全。但是, 人工检查不但耗费了巨大的 人力而且不能够实时地保证钢丝绳的安全; 同时, 强制性定期地更换钢丝绳会产生巨大 的浪费 ${ }^{[3]}$ 。因此, 对钢丝绳的安全状况进行 在线评价是刻不容缓的。另一方面, 工程中 使用的钢丝绳类型广泛、数目巨大, 人工进 行信息管理耗时、耗力, 易出现错误, 不易 于保存。因此利用计算机在数据处理上的优 势, 对钢丝绳的信息进行存储和管理, 有利 于向高效、自动化、智能化方向发展。基于 上述需求, 开发出合理的钢丝绳安全评价及 管理系统是非常必要的。

\section{2. 系统分析和总体设计}

\section{1 需求分析}

通过调查企业内部对钢丝绳安全评价 的需求以及钢丝绳信息的管理方式并进行 仔细分析, 要求系统具有以下功能:

[1] 要求系统具有良好的人机交互界 面，便于操作人员的操作;

[2] 完全人性化设计, 无需专业人士指 导, 即可操作本系统;

[3] 利用声发射技术对钢丝绳安全进 行评价和安全预警;
[4] 规范、完善的钢丝绳基础信息;

[5] 方便的、全方位的数据查询、修改 功能;

[6] 对查询到的记录进行打印;

[7] 系统具有数据备份及数据还原功 能, 能够保证系统数据的安全性;

[8] 用户可自行修改密码。

\section{2 可行性分析}

本系统的开发主要包括对钢丝绳运行 状况进行安全评价及预警和对数据库中的 数据进行操作。考虑到系统的功能要求, 在 系统的技术实现上可以使用 Visual Basic 6.0 开发工具与Microsoft SQL Server 2000 相结合进行软件开发。Visual Basic 6.0组件 功能强大, 可以实现系统的各项功能操作, 并提高开发软件的效率和软件安全性。同 时, 微软提供的SQL Server 2000具有较高的 数据安全性与可维护性, 本系统在投入使用 后, 后期数据维护比较容易, 因此, 本系统 的开发是可行的。

\section{3 总体设计}

钢丝绳安全评价及管理系统主要由登 录、在线评价、钢丝绳信息管理、用户管理、 数据安全、软件信息、帮助等模块组成。

(1) 登录模块

该模块主要用于对登录系统的用户进 行安全性检查, 以防止非法用户进入系统。

（2）在线评价模块

该模块基于声发射检测技术, 对钢丝绳 的运行状况进行安全评价和预警。

（3）钢丝绳信息管理模块

该模块主要用于钢丝绳基本信息的存 储、修改、删除、增加、查询、打印、等操 作。

（4）用户管理模块

该模块主要用于对用户信息的管理以 及用户自行修改密码。

（5）数据安全模块

该模块用于对数据库进行数据备份与 恢复等操作。 
（6）软件信息模块

该模块包括软件说明及关于系统信息。

(7) 帮助

该模块主要对初次使用系统的用户给 出操作的帮助信息。

系统的功能结构如图1所示。

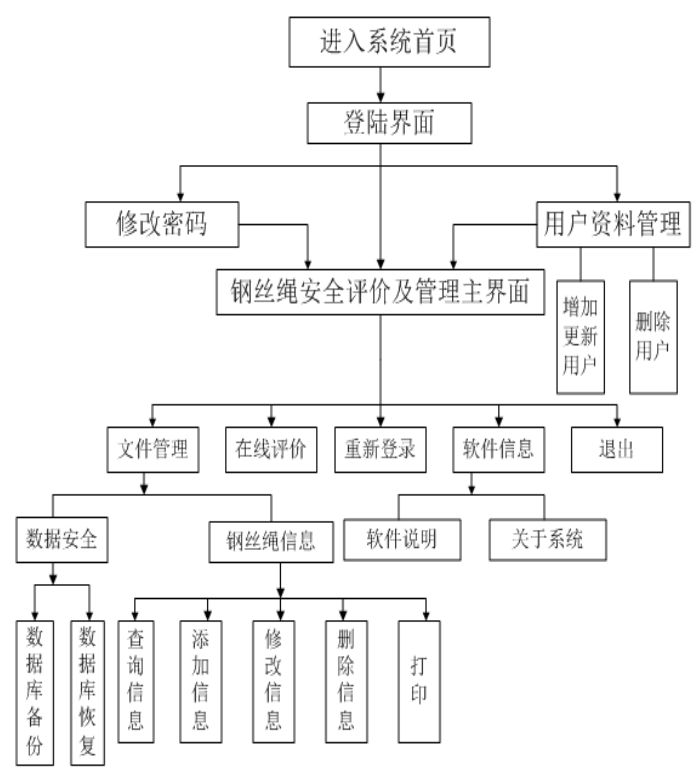

图1 系统功能结构图

\section{3. 系统实现}

\section{1 系统数据库设计}

选择Microsoft SQL Server 2000数据库 管理系统为系统运行提供后台服务, 数据库 的名称为 “钢丝绳信息”, 数据库中包含钢 丝绳信息表、用户信息表。其中, 钢丝绳信 息表用于保存钢丝绳的型号、股数等基本信 息, 同时为钢丝绳安全评价提供数据支持, 其中不允许为空的为钢丝绳型号。用户信息 表用于保存用户的基本信息。

\section{2 系统登录}

启动系统后, 首先弹出欢迎界面, 点击 “欢迎进入系统” 按钮后, 弹出系统登录窗 口。系统登录主要用于对登录系统的用户进 行安全性检查, 以防止非法用户进入该系 统。只有合法的用户才可以登录系统, 如果 用户的用户名或密码错误, 系统给出相应的
提示。在界面的状态栏中显示操作员信息及 系统时间。系统登录界面如图2所示。

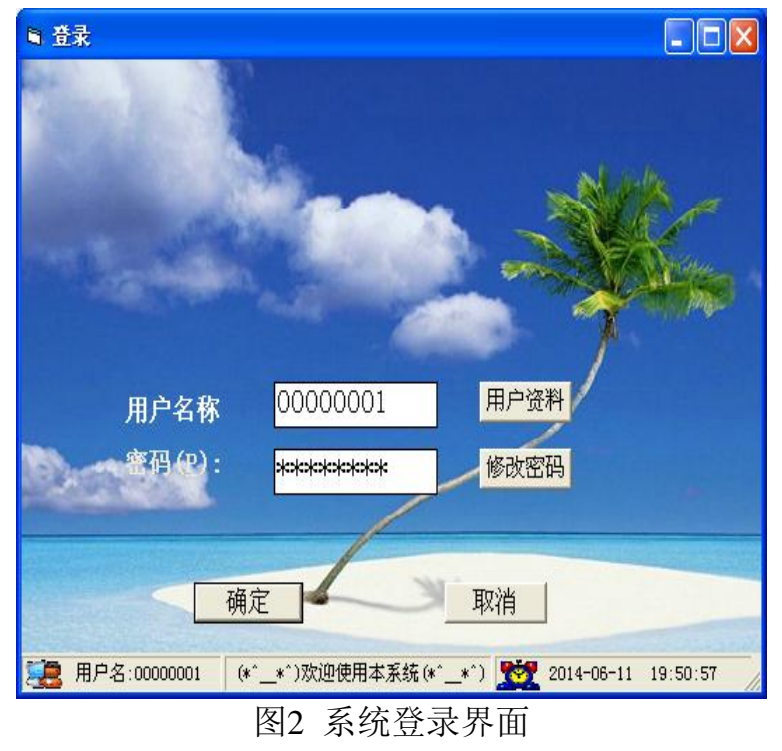

\section{3 在线评价}

声发射作为一种先进的无损检测技术， 能够有效的检查出材料损伤过程所发出的 瞬态弹性波, 如裂纹扩展、纤维断裂等。因 此利用声发射技术可以完成对各种产品和 结构中可能发生的缺陷、故障进行预报, 以 保障其安全运行, 在设备的在线监测中具有 重要的地位。在多年的生产实践和研究中发 展起来的声发射参数分析法是对信号处理 的一种经典分析法, 目前仍然在声发射检测 中被广泛使用, 并已被证明在多数情况下可 以解决工程实践中的问题。对声发射信号进 行频域谱分析作为重要的辅助分析手段, 可 以更好地判断缺陷类型。

在线评价模块基于声发射检测手段, 利 用声发射信号的参数分析法结合频域谱分 析法对钢丝绳声发射信号进行处理与分析, 实现对钢丝绳断丝率的计算以及对钢丝绳 运行状况的安全评价与预警功能。在线评价 模块建立在大量实践经验上, 直观易操作, 实现了对钢丝绳声发射断丝信号的判断并 且为进行安全评价提供了依据。安全评价以 及预警功能的实现，极大地方便了对声发射 源特性的考察, 并且实现了对缺陷的实际有 害程度的评估, 便于及时制定检修计划, 从 而实现在工程应用中有效预报出潜在危险, 降低事故发生可能性以及维修成本。由于本 软件界面设计采用VB 6.0, 使得系统界面清 晰直观，易于操作。 
用户首先选择钢丝绳型号, 该型号钢丝 绳的参数为下一步进行的安全评价提供数 据支持; 接着输入幅值、绝对能量、频谱范 围等参数, 利用参数分析法结合频域谱分析 进行参数处理, 界面显示断丝率, 并对钢丝 绳运行状况进行安全评价给出可能的预警。 运行结果如图3所示。

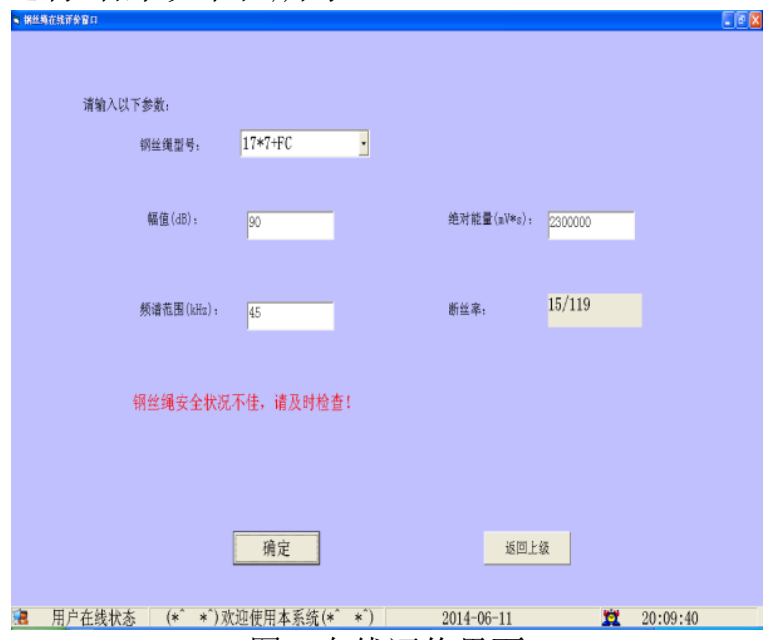

图3 在线评价界面

\section{4 钢丝绳信息管理}

钢丝绳信息管理模块主要用于对钢丝 绳基本信息的存储、修改、删除、增加、查 询、打印、等操作。钢丝绳信息表中储存了 钢丝绳的型号、股数、股钢丝数、形式、绳 芯、捻距、直径、单丝直径、制造厂商、生 产日期信息。用户可以通过钢丝绳股数、捻 距、直径等字段查询相关记录, 同时, 可以 对所有记录进行修改、删除、增加及打印等 操作。其原理为: 利用VB的adodc控件连接 数据库, 同时作为datagrid控件的数据源来 显示所有记录; 另一方面利用SQL语言中的 查询、添加、删除、修改等语句来实现相应 的功能。界面设计简单明了, 主要包括状态 栏、工作区, 为用户提供了便捷的访问路径。 其中, 状态栏显示系统运行时间和用户信 息, 工作区内显示了所有的信息记录及对应 的功能按钮。钢丝绳信息管理界面如图4所 示。

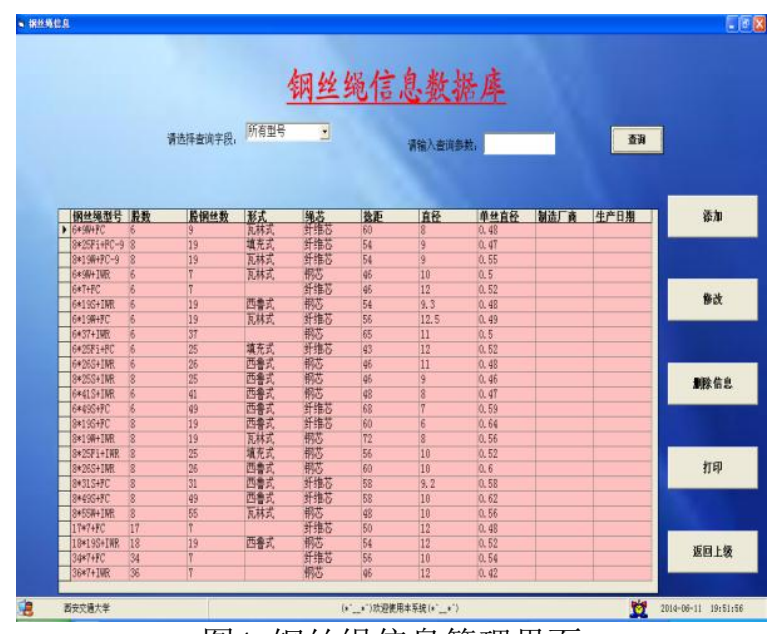

图4 钢丝绳信息管理界面

\section{5 用户管理}

用户管理模块主要由用户信息、密码修 改组成。用户信息中包含了用户的用户名、 密码、资格等信息, 为了对私人信息进行保 护，只有拥有权限的用户可以进入该模块， 同时系统提供相应的查询、修改、增加、删 除功能; 所有用户可在登录成功后对其密码 进行修改, 系统要求用户两次输入新密码, 只有在两次输入密码相同的情况下才可以 完成修改。用户信息界面如图5所示。

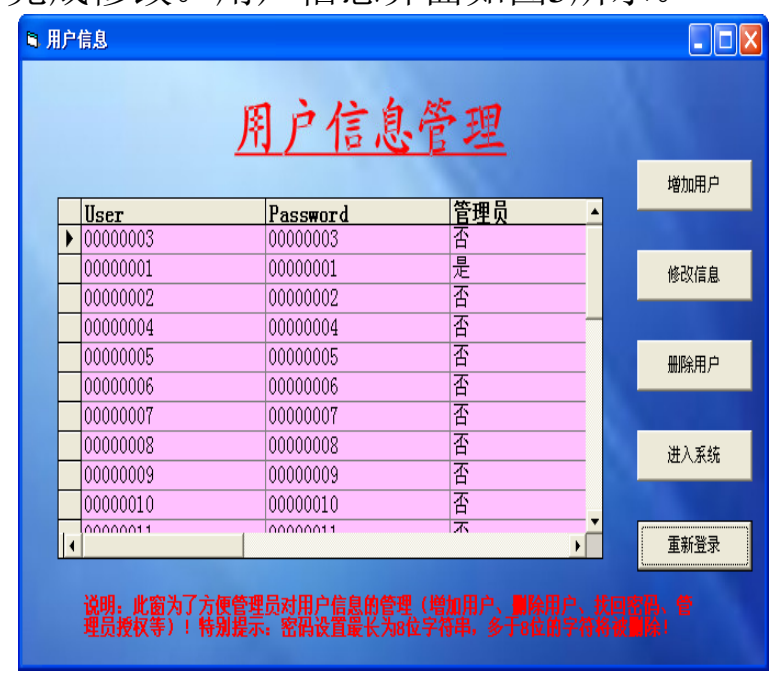

图5 用户信息界面

\section{6 数据安全}

数据安全模块主要由数据库备份与恢 复组成。为了防止意外事件发生, 如病毒、 硬盘的损坏或突然掉电等, 管理员需要经常 做好数据库的备份, 就是把当前的数据库文 件复制到另一个目录。数据库恢复是数据库 备份的逆操作, 就是把备份的数据库文件覆 盖到当前目录。数据库备份与恢复界面如图 6所示。 


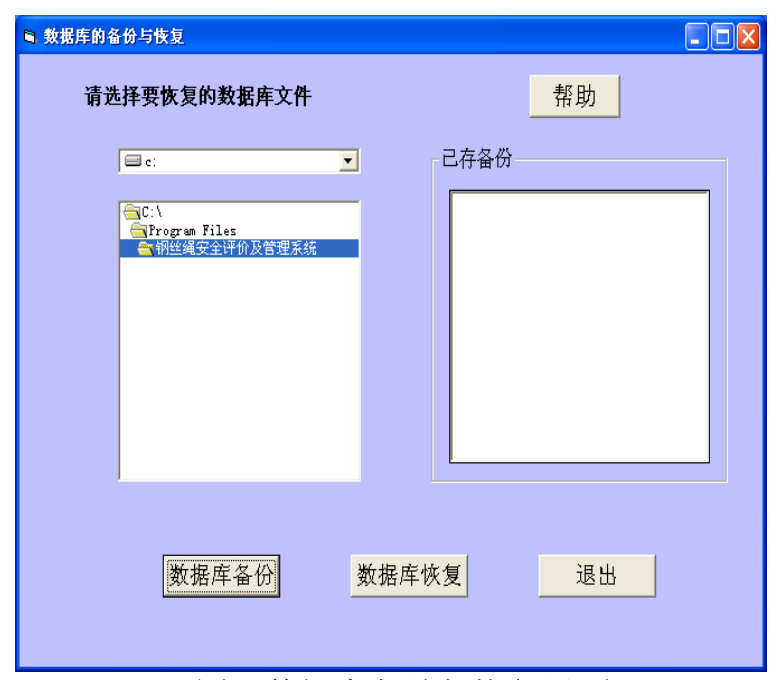

图6 数据库备份与恢复界面

\section{7 其他模块}

软件信息模块包括软件说明及关于系 统信息, 其中 “软件说明” 主要介绍系统开 发的依据标准以及系统需求; “关于系统” 主要介绍系统的基本信息。帮助模块用于对 初次使用的用户给出帮助信息, 利于操作者 更好的使用。“重新登录” 只是在用户已经 登录的情况下, 重新返回登录模块, 主要用 于用户的切换。

\section{4. 结束语}

(1) 本文针对钢丝绳运行中对安全及 信息管理的实际需要, 建立了一个实用的钢 丝绳安全评价及管理系统, 通过声发射检测 法实现对钢丝绳断丝信号的判断, 并给出运 行状况的安全评价及预警; 同时, 实现了对 钢丝绳信息、用户信息的存储, 并且提供查 询、修改、打印等功能。

（2）通过为用户分配权限来保证数据 的一致性、保密性; 数据库的备份与恢复保 证数据的安全可靠。

(3) 系统采用良好地人机对话模式, 界面设计友好, 使操作者无需具备专业的计 算机知识, 便可完成所有操作。

(4) 系统在Windows XP及Windows 7 (x86)平台下进行测试后, 可以稳定运行, 同时, 满足对钢丝绳运行状况安全评价及管 理工作的需要。

\section{致谢}

本文为陕西省科学技术研究发展计划 资助项目《钢丝绳损伤演化评价方法研究及 在线监测系统开发》(2012K06-22)的阶段性 成果之一。

\section{References}

[1] Sun Guilin, Yuan Hualin. Hoisting and mechanical safety engineering[M]. Beijing: Beijing Academy of Economics Press, 1992 : 5-10.

[2] Hua Wenyue. Safety use and discarding as useless of wire rope for engineering machine[J]. Steel Wire Products, 2007, 33(5) : 73-77.

[3] Wu Shuhui. The study of the testing system of the loss of the metallic cross-sectional area of wire rope[D]. Wuhan: Wuhan University of Technology, 2004: 11-14.

[4] Cao Yinni, Zhang Donglai, Xu Dianguo. The stste-of art of quantitative nondestructive testing of wire ropes[J]. Nondestructive Testing, 2005, 27(2): 91-95.

[5] He Chundong. Theoretical discuss and developing study on steel wire rope flaw detection[D]. Liaoning: Liaoning Technical University, 2005: 6-8, 13-15.

[6] Shen Gongtian, Gen Rongsheng, Liu Shifeng. Parameter analysis of acoustic emission signals[J]. Nondestructive Testing, 2002, 24(2):72-77.

[7] Nie Lin, Qian Xiaoyao, Yuan Changming, Zhang Weigang. Simulation of wire rope stretching process and feature analysis of the acoustic emission on the load of 10 $\mathrm{kN}[\mathrm{J}]$. Journal of China Jiliang University, 2013,24(1): 86-91.

[8] Gen RongSheng, Shen Gongtian, Liu Shifeng. Acoustic emission signal processing technique based on waveform analysis[J]. Nondestructive Testing, 24(6): 257-261. 